\title{
Influência de Variedades de Cana-de-Açúcar, Incorporadas em Dieta Artificial, no Desenvolvimento de Diatraea saccharalis (Fabr.) e no seu Parasitismo por Cotesia flavipes (Cam.).
}

\author{
Arlindo L. Boiça Jr. ${ }^{1}$, Fernando M. Lara ${ }^{1}$ e Maria P. Bellodi \\ ${ }^{1}$ Departamento de Entomologia e Nematologia, FCAV/UNESP, Rodovia \\ Carlos Tonanni, Km 5, 14870-000, Jaboticabal, SP.
}

An. Soc. Entomol. Brasil 26(3): 537-542 (1997)

Influence of Stem of Sugarcane Varieties the Development of Diatraea saccharalis (Fabr.) and its Parasitism by Cotesia flavipes (Com.).

\begin{abstract}
In this study it was observed the influence of two sugarcane (Saccharum spp.) varieties, that were included in an artificial diet as powdered stem, on development of Diatraea saccharalis (Fabr.) and parasistism by Cotesia flavipes (Cam.). The variety SP71 - 1081 was less suitable $(\mathrm{P} \leq 0.05)$ to $D$. saccharalis development than SP 71-3146, delaying larval and pupal period and decreasing larval weight; no significant differences $(P>0.05)$ were observed for larval and pupal survival as well as pupal weight. D. saccharalis larvae reared on artificial diet and on sugarcane stem were equally preferred by the parasitoid $C$. flavipes. Larvae reared on artificial diet with powdered stem of the tested varieties promoted a similar development of $C$. flavipes. The percent of parasitism in the larvae reared in artificials diets contention of the SP 71$1081(84.0 \%)$ and SP 71-3146 (81.3\%) varieties were similar.
\end{abstract}

KEY WORDS: Insecta, sugarcane borer, plant resistance, biological control, extrinsic resistance.

RESUMO - Verificou-se a influência de duas variedades de cana-de-açúcar (Saccharum spp.), incorparados em dieta artificial, na forma de colmos triturados, no desenvolvimento de Diatraea saccharalis (Fabr.) e no parasitismo de Cotesia flavipes (Cam.). A variedade SP71-1081 foi menos adequada ao desenvolvimento de D. saccharalis comparada à SP71-3146, aumentando os períodos larval e pupal (dias) e, diminuindo o peso larval; não houve diferenças para as viabilidades larval e pupal e peso de pupa. Lagartas oriundas das dietas artificiais e lagartas alojadas no interior dos toletes das variedades de cana, foram igualmente parasitadas por $C$. flavipes. Lagartas criadas em dieta artificial contendo colmos triturados das variedades testadas, promoveram desenvolvimento semelhante de $C$. flavipes. A percentagem de parasitismo foi semelhante nas larvas criadas em dietas artificiais contendo colmos das variedades SP 71 - $1081(84,0 \%)$ e SP 71-3146 (81,3\%).

PALAVRAS-CHAVE: Insecta, resistência de plantas, controle biológico, resistência extrínseca. 
A cultura da cana-de-açucar (Saccharum spp.), devido sua importância para a economia nacional, expandiu-se, praticamente, por todos os estados brasileiros. Apesar da rusticidade dessa cultura, ela defronta-se com uma série de problemas agronômicos, incluindo a incidência de pragas e, em particular, a presença da broca Diatraea saccharalis (Fabr.) (Lepidoptera: Pyralidae), considerada uma das mais importantes na região de Jaboticabal (Lara et al. 1980).

Com relação aos danos, Guagliumi (1972/ 73) e Gallo et al. (1988) referiram que as lagartas se alimentam nos primeiros dias dos tecidos foliares, penetrando, em seguida, no interior dos colmos. Seus prejuízos diretos originam-se das galerias que fazem nos colmos, provocando perda de peso e tombamento da planta pelo vento, entre outros. Indiretamente, favorecem a penetração de fungos nas plantas, causando a podridão vermelha do colmo, levando à inversão da sacarose e diminuição da produção de álcool.

Dentre os métodos de controle mais estudados, encontram-se o biológico e o uso de variedades resistentes. Assim, com relação ao primeiro, Souza (1961), Moraes \& Berti Filho (1974) e Gallo et al. (1988) ressaltaram o seu emprego, sendo apresentado por este último autor, o processo de criação de Cotesia flavipes (Cam.), todo o esquema de liberação, dispersão e a eficiência no controle da broca da cana-de-açucar. Quanto a variedades resistentes, diversos trabalhos foram realizados no Brasil, como os de Gallo (1954), Amaral \& Arruda (1964) e Macedo et al. (1977). Derneika \& Lara (1991) concluíram que as variedades SP71-345, SP71-6113 e SP71-1081 comportaram-se como moderadamente resistentes à broca, enquanto CP51-22, SP71-3146 e SP71-5574 foram altamente suscetíveis.

O uso de variedades resistentes apresenta inúmeras vantagens, entre elas a de que a planta resistente é geralmente compatível com a aplicação de outras táticas de manejo do inseto, incluíndo o controle biológico. Todavia, em certos casos, a resistência a insetos e o controle biológico podem ser antagonistas e é importante que sua interação seja bem estudada antes dessas táticas serem implementadas a campo. Exemplos de resistência extrínseca e de inter-relações negativas foram observados por Boethel \& Eikenbary (1986) e Lara (1991).

Neste trabalho observou-se a influência de colmo seco triturado de duas variedades de cana-de-açúcar, incorporados à dieta artificial, no desenvolvimento de $D$. saccharalis e no seu parasitismo por $C$. flavipes.

\section{Material e Métodos}

Os experimentos foram conduzidos no período de novembro de 1992 a janeiro de 1993, coletando-se colmos de duas variedades de cana-de-açucar, SP71-1081 (moderadamente resistente) e SP71-3146 (suscetível), das quais retirou-se a parte central do colmo, que foi seca, triturada e incorporada em dieta artificial à base de feijão, semelhante àquela relatada por De Bortoli et al. (1989).

Biologia de $\boldsymbol{D}$. saccharalis. Tubos de vidro $(8,5 \mathrm{~cm} \times 2,5 \mathrm{~cm})$ contendo dieta foram inoculados, individualmente, com cinco lagartas recém eclodidas, sendo posteriormente tamponados com chumaços de algodão hidrófugos, utilizando-se 25 tubos para cada variedade. Após a inoculação, os recipientes foram mantidos a $29 \pm 1{ }^{\circ} \mathrm{C}, 60 \pm 10 \%$ de UR e fotofase de 14 horas. A seguir, iniciaram-se as observações diárias, verificando-se o período larval de $D$. saccharalis, peso de lagartas aos 14 dias de idade, viabilidade larval, período pupal, peso de pupas com 24 horas de idade e viabilidade pupal.

Atratividade de $C$. flavipes por lagartas de D. saccharalis. Utilizaram-se placas de Petri (7 cm x 1,5 cm) com duas lagartas da broca com 19 dias de idade, oriundas cada uma de criação correspondente em cada variedade. A seguir liberaram-se duas fêmeas do parasitóide (teste com chance de escolha); todo o conjunto foi repetido por 50 vezes. Em outro ensaio, as lagartas de 19 dias de idade, criadas na dieta artificial, relatada 
anteriormente, foram mantidas temporariamente no interior do tolete de cana da respectiva variedade, por 24 horas, para que o odor se tornasse mais natural. Da mesma forma que no ensaio anterior, foram utilizadas duas fêmeas do parasitóide por placa de Petri. Em ambos os ensaios, foram realizadas observações do número de oviposições do parasitóide nas lagartas oriundas de cada variedade, em períodos de um, três e cinco minutos.

Biologia de $C$. flavipes em lagartas de $D$. saccharalis. As lagartas criadas nas dietas contendo colmo seco triturado das duas variedades de cana foram oferecidas, aos 19 dias de idade, para oviposição por $C$. flavipes, permanecendo, a seguir, uma lagarta dentro de cada caixa plástica $(2 \mathrm{~cm} \times 6 \mathrm{~cm})$ contendo as respectivas dietas. Foram realizadas 150 repetições. Foram avaliados a duração de período de ovo a pupa (dias), o peso de pupas (mg), a viabilidade pupal, o número de parasitóides emergidos e a percentagem de parasitismo. Todos os parâmetros avaliados foram submetidos à análise de variância, pelo teste $\mathrm{F}$, e as médias comparadas pelo teste de Tukey, a $5 \%$ de probabilidade.

\section{Resultados e Discussão}

Biologia de $D$. saccharalis. Foram observadas diferenças estatísticas significativas $(\mathrm{P} \leq 0,05)$ para a duração e peso larval e duração da fase pupal, constatandose maior duração dessas fases e menor peso de lagartas, em dietas contendo colmo da variedade resistente SP71-1081, sugerindo que esse material apresenta não preferência para alimentação e/ou antibiose (Tabela 1). Ao contrário, nos indivíduos criados em dieta contendo colmo da variedade SP71-3146, as fases larval e pupal foram mais rápidas e as lagartas mais pesadas, evidenciando um favorecimento no desenvolvimento da broca, constituíndo-se, portanto, em material suscetível, concordando com os resultados observados em campo por Derneika \& Lara (1991).

\section{Atratividade de $C$. flavipes por Lagartas} de $D$. saccharalis. Em todas as avaliações efetuadas com a dieta artificial não foram detectadas diferenças significativas $(\mathrm{P}>0,05)$ entre as variedades utlilizadas, ocorrendo o mesmo quando as lagartas foram mantidas por 24 horas no colmo de cada variedade, após 19 dias na respectiva dieta (Fig. 1). Esses resultados mostram que as lagartas criadas nas duas variedades fornecem estímulos semelhantes de atratividade a C. flavipes, o que significa que nenhum dos materiais apresenta resistência extrínseca e que a variedade moderadamente resistente (SP 71 1081) não age adversamente sobre o parasitóide, o que é benéfico quanto à integração das duas táticas de controle. Outro fato observado foi que a maior oviposição do

Tabela 1 - Média ( \pm EP) da duração, peso e viabilidade das fases larval e pupal de Diatraea saccharalis, criada em dieta artificial contendo colmo seco triturado de duas variedades de cana-de-açúcar.

\begin{tabular}{lccccccc}
\hline & \multicolumn{3}{c}{ Fase Larval $^{1}$} & & \multicolumn{3}{c}{ Fase Pupal $^{1}$} \\
\cline { 2 - 4 } Variedades & $\begin{array}{c}\text { Duração } \\
\text { (dias) }\end{array}$ & $\begin{array}{c}\text { Peso de } \\
\text { lagartas } \\
(\mathrm{mg})\end{array}$ & $\begin{array}{c}\text { Viabilidade } \\
(\%)\end{array}$ & & $\begin{array}{c}\text { Duração } \\
(\text { dias })\end{array}$ & $\begin{array}{c}\text { Peso de } \\
\text { Pupas } \\
(\mathrm{mg})\end{array}$ & $\begin{array}{c}\text { Viabilidade } \\
(\%)\end{array}$ \\
\hline SP 71-1081 & $27,9 \pm 3,45 \mathrm{a}$ & $24,5 \pm 1,78 \mathrm{a}$ & $64,8 \pm 5,85 \mathrm{a}$ & & $1,1 \pm 0,29 \mathrm{a}$ & $120,7 \pm 4,49 \mathrm{a}$ & $65,7 \pm 7,08 \mathrm{a}$ \\
SP 71-3146 & $26,8 \pm 2,97 \mathrm{~b}$ & $30,4 \pm 1,69 \mathrm{~b}$ & $69,60 \pm 4,62 \mathrm{a}$ & & $9,2 \pm 0,21 \mathrm{~b}$ & $127,8 \pm 4,28 \mathrm{a}$ & $69,9 \pm 5,81 \mathrm{a}$ \\
\hline
\end{tabular}

${ }^{1}$ Médias seguidas de mesma letra não diferem estatisticamente pelo teste de Tukey, ao nível de $5 \%$ de probabilidade. 


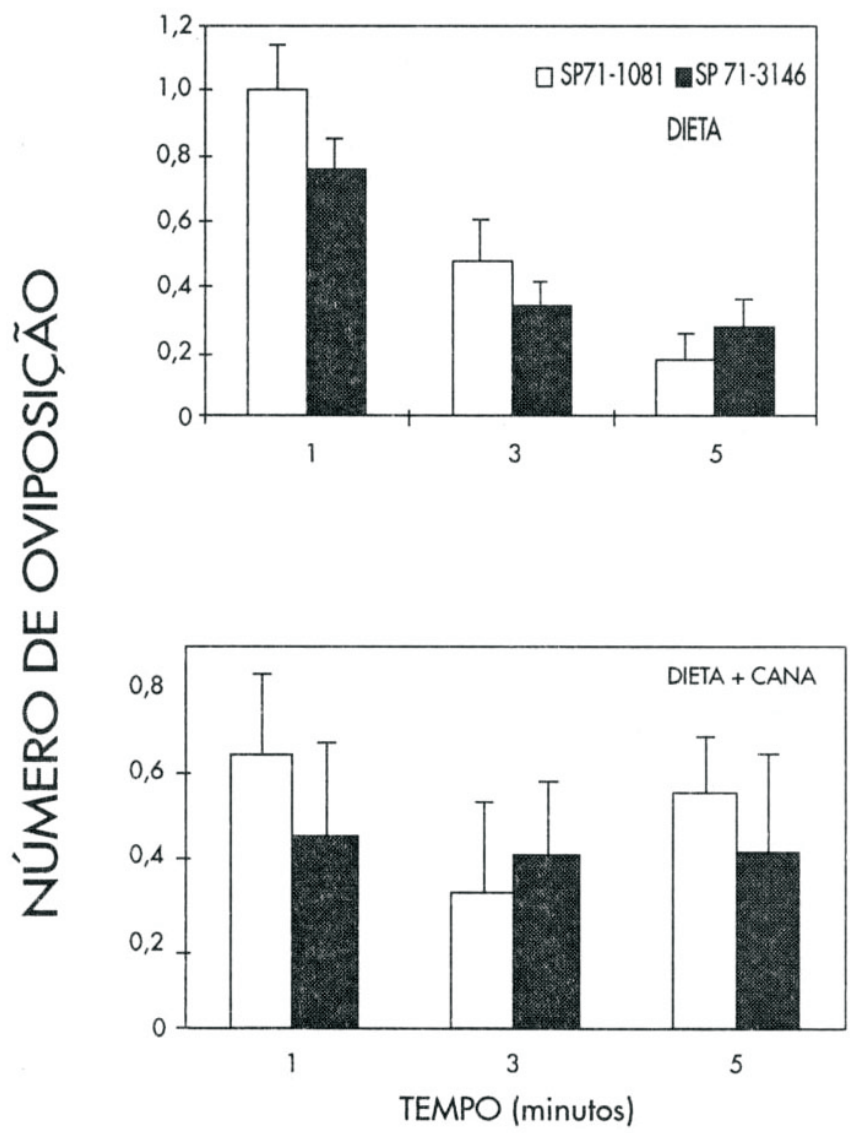

Figura 1. Média ( \pm EP) do número de oviposições de Cotesia flavipes a 1,3 e 5 minutos da liberação, em lagartas de Diatraea saccharalis criadas em dietas artificiais contendo colmo seco triturado (Dieta), e alojados temporariamente em toletes (Dieta + Cana) de duas variedades de cana-de-açúcar (não houve diferenças estatísticas significativas entre os tratamentos, dentro dos tempos estudados, $\mathrm{P}>0,05)$.

parasitóide ocorreu logo no primeiro minuto após sua liberação, decrescendo para os demais períodos. É interessante salientar que, com a secagem e trituração do colmo de canade-açucar, muitos fatores físicos, químicos e morfológicos de atratividade ou repelência das plantas foram eliminados, o que poderia modificar esses resultados em condições de campo. Tal fato pode ser verificado nas observações de Boethel \& Eikenbary (1986) onde comentam que os parasitóides orientamse inicialmente em resposta aos estímulos fornecidos pela planta, e que numa segunda etapa, secundariamente, respondem aos estímulos fornecidos pelo hospedeiro. Lara (1991) comenta que estes estímulos da planta podem ser provocados por produtos químicos, cairomônios, ou fatores morfológicos, ressaltando que esses últimos são tão importantes e diversificados quanto os primeiros, porém menos estudados. 
Tabela 2. Média ( \pm EP) da duração dos período de ovo a pupa; peso e viabilidade pupal, número de insetos emergidos, percentagem de parasitismo de Cotesia flavipes, em lagartas de Diatraea saccharalis criadas em dieta artificial contendo colmo seco triturado de duas variedades de cana-de-açúcar.

\begin{tabular}{|c|c|c|c|c|c|c|}
\hline \multirow[t]{2}{*}{ Variedades } & \multirow{2}{*}{$\begin{array}{l}\text { Duração do Período } \\
\text { de ovo } \\
\text { a pupa (dias) }{ }^{1}\end{array}$} & \multicolumn{3}{|c|}{ Fase Pupal ${ }^{1}$} & \multirow{2}{*}{$\begin{array}{c}\mathrm{N}^{\mathrm{o}} \text { de Adultos } \\
\text { Emergidos }^{1}\end{array}$} & \multirow{2}{*}{$\begin{array}{c}\text { Percentagem de } \\
\text { Parasitismo }^{1}\end{array}$} \\
\hline & & $\begin{array}{c}\text { Duração } \\
\text { (dias) }\end{array}$ & $\begin{array}{l}\text { Peso de Pupas } \\
\text { (mg) }\end{array}$ & $\begin{array}{c}\text { Viabilidade } \\
(\%)\end{array}$ & & \\
\hline SP 71-1081 & $12,6 \pm 0,07 a$ & $7,9 \pm 0,04 a$ & $65,7 \pm 2,04 a$ & $72,3 \pm 2,39 a$ & a $\quad 65,2 \pm 3,91 \mathrm{a}$ & a $84,0 \pm 3,02 \mathrm{a}$ \\
\hline SP 71-3146 & $12,6 \pm 0,06 \mathrm{a}$ & $7,8 \pm 0,05 \mathrm{a}$ & $66,4 \pm 2,14 a$ & $77,7 \pm 5,03 \mathrm{a}$ & a $67,9 \pm 3,73 \mathrm{a}$ & a $81,3 \pm 3,20$ a \\
\hline
\end{tabular}

${ }^{1}$ Médias seguidas da mesma letra não diferem estatisticamente pelo teste de Tukey, ao nível de $5 \%$ de probabilidade.

Biologia de $C$. flavipes em lagartas de $D$. saccharalis. Para os parâmetros avaliados, não ocorreram diferenças estatísticas significativas entre as variedades testadas (Tabela 2). Tal fato evidência que as lagartas de D. saccharalis criadas em dieta artificial, contendo colmo seco triturado das variedades SP71-1081 e SP71-3146 proporcionam desenvolvimento semelhante de $C$. flavipes e que, portanto, a resistência moderada de SP71-1081, não prejudica o parasitóide, como também, constata-se igualdade no nível de parasitismo, o que revela a adequação da resistência com o controle biológico, conforme comentada anteriormente em relação a atratividade.

\section{Agradecimentos}

Ao $\mathrm{CNPq}$ pelas bolsas de produtividade em pesquisa concedidas aos dois primeiros autores.

\section{Literatura Citada}

Amaral, S.F. \& H.V. Arruda. 1964. Grau de suscetibilidade de 15 variedades de cana-de-açucar à broca Diatraea saccharalis (F., 1794) (LepidopteraPyralidae). Arq. Inst. Biol. 31:79-83.

Boethel, D.J. \& R.D. Eikenbary. 1986. Interactions of plant resistance and parasitoids and predators os insects. Chichester, Ellis Horwood, 224p.

De Bortoli, S.A., A.L. Boiça Júnior, H.F. da Cunha, M.G.A. Lendell \& P.P. de Matos. 1989. Influência de diferentes genótipos de sorgo e milho na biologia de Diatraea saccharalis (Fabricius, 1794) (Lepidopetra, Pyralidade), criada em dieta artificial. Ecossistema 14: 204212 .

Derneika, O. \& F.M. Lara. 1991. Resistência de cana-de-açúcar a Diatraea saccharalis (Fabr., 1794) (LepidopteraPyralidae): comportamento de variedades em três cortes e em quatro locais do Estado de São Paulo. An. Soc. Entomol. Brasil 20:359-368.

Gallo, D. 1954. Infestação da broca nas principais variedades de cana. Rev. Agric. 29:149-155.

Gallo, D., O. Nakano, S.S. Neto, R.P.L. Carvalho, G.C. Batista, E. Berti Filho, J.R.P. Parra, R.A. Zucchi, S.B. Alves \& J.D. Vendramim. 1988. Manual de entomologia agrícola. São Paulo, Agronômica Ceres, 649p.

Guagliumi, P. 1972/73. Pragas da cana-deaçúcar no nordeste do Brasil. Rio de 
Janeiro: Instituto do Açúcar e do Álcool. Col. Canav., 622p.

Lara, F.M. 1991. Princípios de resistência de plantas a insetos. São Paulo, Ed. Ícone, 336.

Lara, F.M., G.C. Barbosa $F^{\circ} \&$ J.C. Barbosa. 1980. Danos acarretados por Diatraea saccharalis (Fabricius, 1794) na produção de sorgo granífero. Científica 8:105-111.

Macedo, N., A.C. Mendes \& P.S.M.

Botelho. 1977. Susceptibility of the main commercial sugarcane varieties to the moth borer, Diatraea saccharalis (Fabr., 1974) in the Southern-Central Brazil, p.693-701. In Proc. Congr. Int. Soc. Sug. Cane Tech, 16, São Paulo.

Moraes, G.J. de \& E. Berti Filho. 1974. Coleobrocas que ocorrem em essências florestais. Inst. Pesq. Eng. Flor., Piracicaba, 9:27-42.

Souza, H.D. 1961. Dois parasitos de ovos da broca da cana (Diatraea sacharalis) (Fabr., 1794). Divulgação Agr. 3:2-5.

Recebido em 29/08/96. Aceito em 27/10/97. 